Results 109 patients (43\% men; 57\% women) met inclusion criteria. Mean age was $54 \pm 13.5$ years and mean BMI $26.5 \pm 4.8 \mathrm{~kg} / \mathrm{m}^{2}$. $58.7 \%$ had Rheumatoid Arthritis, 19.3\% Ankylosing Spondylitis, 1.8\% Juvenile Idiopathic Arthritis, 16.5\% Psoriatic Arthritis and $3.7 \%$ Psoriasis. $82 \%$ self-administrated the pen, and $71 \%$ the syringe. The median pain with the syringe was 3 [interquartile range (IOR): $2-6]$ and with the pen was 4 [IOR: $2-5$ ] $(\mathrm{P}=0.008)$. 65\% reported the same pain with both devices. $35 \%$ reported differences in pain and most of them $(71 \%)$ had much pain $(>5)$ with the pen and little pain $(<5)$ with the syringe.

There was a statistically significant association of pain with gender: women had more pain with the pen $(P=0.03)$, but less with the syringe $(p>0.05)$. There was no association with BMI, age or diagnosis. $59 \%$ preferred the pen, $25 \%$ the syringe, and $16 \%$ did not mind.

Conclusions An association of pain with pen device and female gender was found. However there was no association with BMI, age or diagnosis. Acceptance of the pen and self-administration were higher even though pain was greater, so it is necessary to maintain both devices to assure adherence.

No conflict of interest.

\section{OHP-038 EVALUATION OF QUALITY OF LIFE IN PATIENTS WITH MULTIPLE SCLEROSIS}

doi:10.1136/ejhpharm-2013-000276.412

'MC Izquierdo Navarro, 'C Matallana Martin, 'V Martínez Santana, ${ }^{2 M A}$ Calleja Hernandez, 'AM López González, 'MT Sánchez Sánchez. 'Hospital Clínico Universitario, Pharmacy Department, Valladolid, Spain; ${ }^{2}$ Hospital Vírgen de la Nieves, Pharmacy Department, Granada, Spain

Background Several studies have evaluated quality of life (QoL) by filling in the EuroQoL-5D..In most of them, it is found that the two dimensions of EuroQoL-5D most associated with a poor QoL are pain/discomfort and anxiety/depression.

Purpose To find the dimensions of EuroQoL-5D that are more frequently associated with $\mathrm{QoL}$ in patients diagnosed with RelapsingRemitting Multiple Sclerosis (RRMS).

Materials and Methods Observational, four-month, cross-sectional study (January-April 2012) to assess QoL in patients diagnosed with RRMS.

Sex, age and Expanded Disability Status Scale (EDSS) were gathered from Pacientes Externos (Farmatools programme 2.4 version).

Patients who filled in the EuroQoL-5D returned it to the pharmacy service.

Results 84 patients were included; 62 completed the questionnaire.

Mean age was $36.94 \pm 8.67 .65 .47 \%$ of patients were women, $34.52 \%$ were men. The mean EDSS was $2.03 \pm 1.50$.

The survey results of the questionnaire broken down by items were:

\section{Abstract OHP-038 Table 1}

\begin{tabular}{llll}
\hline & & \multicolumn{2}{c}{ Number } \\
\hline Mobility & I have no problems walking & 42 & 67.7 \\
& I have some problems & 20 & 32.3 \\
Personal Care & I am confined to bed & 0 & 0 \\
& I have no problems with self-care & 56 & 90.3 \\
& I have some problems & 6 & 9.7 \\
Usual activities & I am unable to wash or dress myself & 0 & 0 \\
& I have no problems with performing my usual activities & 42 & 67.7 \\
Pain/discomfort (P/D) & I have some problems & 20 & 32.3 \\
& I am unable to perform my usual activities & 0 & 0 \\
Anxiety/Depression & I have no P/D & 28 & 45.2 \\
& I have moderate P/D & 33 & 53.2 \\
& I have extreme P/D & 1 & 1.6 \\
& I am mot anxious or depressed (A/De) & 28 & 45.2 \\
& I am extremely A/De & 28 & 45.2 \\
\hline
\end{tabular}

The mean value obtained in the questionnaire was $0.71 \pm 0.19$. Conclusions As has been shown in previous studies, the two dimensions of EuroQoL-5D that most affected the QoL were pain/ discomfort and anxiety/depression.

No conflict of interest.

\section{OHP-039 EXPANDING THE INVOLVEMENT OF PHARMACY SERVICES VIA COMPUTERISED MEDICAL FILES}

doi:10.1136/ejhpharm-2013-000276.413

${ }^{1} \mathrm{~N}$ Mansur, ${ }^{1} \mathrm{~T}$ Gruenewald, ${ }^{2} \mathrm{E}$ Sporta, ${ }^{3} \mathrm{M}$ Shindler, ${ }^{3} \mathrm{D}$ Lavi. ${ }^{1}$ Beilinson Hospital, Pharmacy services, Tel Aviv, Israel; 'Beilinson Hospital, Computer Services/Elad Software Systems, Tel Aviv, Israel; ${ }^{3 B}$ Beilinson Hospital, Computer Services, Tel Aviv, Israel

Background Pharmacists are essential for the safe use of medicines, and have a very important role in providing comprehensive drug management. Their crucial responsibilities in medicines management and promoting quality control necessitate developing a computerised tool to improve their communication with other medical team members.

Purpose To develop a pharmacist interface, as a part of the computerised medical file 'Chameleon', to display all the information required by pharmacists for preparing and documenting their intervention.

\section{Materials and Methods}

Step 1: mapping the processes required for implementation of the system

Step 2: preparing a dedicated tool with two components:

1. A pharmacist interface: a screen designed to show all related data required for a clinical pharmacist to form his opinion regarding the medicinal treatment. The pharmacist intervention is documented in an assigned field 'pharmacist follow up', which is also displayed beside the 'physician follow up' field in the physician interface to save switching screens.

2. The pharmacy services as an advisory ward: the pharmacists' team is defined as an advisory ward that can be invited by the physicians. Requests for advice are displayed in a pharmacist work list.

Results The pharmacist interface was integrated into the "Chameleon' and is used regularly. It is a convenient tool that displays all the information required for a professional pharmacist's opinion, and improves medical team communication by allowing this opinion to be viewed by other staff members. There is an ongoing process of assimilation and dissemination of the computerised availability of pharmacy advisory services. There are two topics in development: (a) physician feedback and reference regarding the pharmacist advice, and (b) the ability to monitor all revised cases.

Conclusions The computerised tool satisfies the pharmacist work process and improves communication with the medical staff. The final tool will generate statistics about its contribution to medical personnel and improve the quality of pharmacy services in this medical care hospital.

No conflict of interest.

\section{OHP-040 FINANCIAL ASSESSMENT OF INTRAVENOUS MIXTURE PREPARATION}

doi:10.1136/ejhpharm-2013-000276.414

M Uriarte, MJ Agustín, V Gimeno, I Larrodé, 0 Pascual, P Palomo, MR Abad. Miguel Servet Hospital, Pharmacy Service, Zaragoza, Spain

Background Intravenous treatment is expensive so we studied two different working options.

Purpose To evaluate the savings that preparing intravenous mixtures centrally in the pharmacy service hypothetically made in 2011 in our hospital. 